This is an electronic reprint of the original article. This reprint may differ from the original in pagination and typographic detail.

\author{
Author(s): Javanainen, Arto; Galloway, Kenneth F.; Nicklaw, Christopher; Bosser, Alexandre; \\ Ferlet-Cavrois, Véronique; Lauenstein, Jean-Marie; Pintacuda, Francesco; Reed, \\ Robert A.; Schrimpf, Ronal D.; Weller, Robert A.; Virtanen, Ari
}

Title: $\quad$ Heavy Ion Induced Degradation in SiC Schottky Diodes : Bias and Energy Deposition Dependence

Year: $\quad 2017$

Version:

Please cite the original version:

Javanainen, A., Galloway, K. F., Nicklaw, C., Bosser, A., Ferlet-Cavrois, V., Lauenstein, J.-M., Pintacuda, F., Reed, R. A., Schrimpf, R. D., Weller, R. A., \& Virtanen, A. (2017). Heavy Ion Induced Degradation in SiC Schottky Diodes : Bias and Energy Deposition Dependence. IEEE Transactions on Nuclear Science, 64(1), 415-420.

https://doi.org/10.1109/TNS.2016.2616921

All material supplied via JYX is protected by copyright and other intellectual property rights, and duplication or sale of all or part of any of the repository collections is not permitted, except that material may be duplicated by you for your research use or educational purposes in electronic or print form. You must obtain permission for any other use. Electronic or print copies may not be offered, whether for sale or otherwise to anyone who is not an authorised user. 


\title{
Heavy Ion Induced Degradation in SiC Schottky Diodes: Bias and Energy Deposition Dependence
}

\author{
Arto Javanainen, Member, IEEE, Kenneth F. Galloway, Fellow, IEEE, Christopher Nicklaw, \\ Alexandre L. Bosser, Student Member, IEEE, Véronique Ferlet-Cavrois, Fellow, IEEE, \\ Jean-Marie Lauenstein, Member, IEEE, Francesco Pintacuda, Robert A. Reed, Fellow, IEEE, \\ Ronald D. Schrimpf, Fellow, IEEE, Robert A. Weller, Senior Member, IEEE, and \\ A. Virtanen, Member, IEEE
}

\begin{abstract}
Experimental results on ion-induced leakage current increase in $\mathbf{4 H}-\mathrm{SiC}$ Schottky power diodes are presented. Monte Carlo and TCAD simulations show that degradation is due to the synergy between applied bias and ion energy deposition. This degradation is possibly related to thermal spot annealing at the metal semiconductor interface. This thermal annealing leads to an inhomogeneity of the Schottky barrier that could be responsible for the increase leakage current as a function of fluence.
\end{abstract}

Index Terms - Current-voltage characteristics, Ion radiation effects, Modeling, Power semiconductor devices, Schottky diodes, Silicon carbide

\section{INTRODUCTION}

$\mathrm{S}_{\mathrm{f} e \mathrm{or}}^{\mathrm{II}}$ ILICON CARBIDE (SiC) devices are of great interest for their possible use in power applications in space. Higher breakdown field and thermal conductivity makes $\mathrm{SiC}$ a very attractive material compared to silicon for power electronics. However, like their silicon counterparts, $\mathrm{SiC}$ power devices (MOSFETs and diodes) are sensitive to particle radiation [1], [2]. The response of $\mathrm{SiC}$ Schottky devices to particle radiation is somewhat different from what typically is observed in

${ }^{\mathrm{T}}$ his work was supported by Walter Ahlström Foundation through the Tutkijat Maailmalle program, the European Space Agency (ESA/ESTEC Contract 4000111630/14/NL/PA) and the Academy of Finland under the Finnish Centre of Excellence Programme 2012-2017 (Project No 2513553, Nuclear and Accelerator Based Physics).

A. Javanainen, A. Bosser and A. Virtanen are with the University of Jyvaskyla, Department of Physics, P.O. Box 35, FI-40014, University of Jyvaskyla, Finland. (email: \{arto.javanainen, alexander.l.bosser, ari.j.virtanen\}@jyu.fi)

A. Javanainen is also with the Electrical Engineering and Computer Science Department, Vanderbilt University, Nashville, TN 37235 USA. (email: arto.javanainen@vanderbilt.edu)

K. Galloway, R. Reed, R. Schrimpf and R. Weller are with the Electrical Engineering and Computer Science Department, Vanderbilt University, Nashville, TN 37235 USA. (email: \{kenneth.f.galloway, robert.reed, ron.schrimpf, robert.a.weller\}@vanderbilt.edu)

C. Nicklaw is with Silvaco, Inc. 4701 Patrick Henry Drive, Bldg. 2, Santa Clara, CA 95054, USA, (email: chris.nicklaw@silvaco.com)

V. Ferlet-Cavrois is with the European Space Agency, ESTEC, 2200 AG Noordwijk, The Netherlands. (email: veronique.ferlet-cavrois@esa.int)

J.-M. Lauenstein is with NASA/GSFC, Code 561.4, Greenbelt, MD 20771 USA. (email: jean.m.lauenstein@nasa.gov)

F. Pintacuda is with the STMicroelectronics Srl, I-95121 Catania, Italy. (email: francesco.pintacuda@st.com) silicon-based power devices. SiC Schottky power diodes exhibit Single Event Burnout (SEB) as silicon-based devices do, but at bias voltages below the SEB threshold, SiC Schottky devices also exhibit gradual degradation under heavy-ion exposure [2], [3]. The gradual degradation makes evaluation of other effects, such as SEB, more difficult.

This work explores the effects of experimental conditions (including device structure, ions and applied biases) on the gradual leakage current increase observed in $\mathrm{SiC}$ Schottky power diodes with increasing heavy-ion fluence. The degradation depends strongly on the ions selected and bias voltages applied during exposure. Monte Carlo simulations indicate that the overall physical structure of the tested diode (e.g., the bonding wires) affects the observed radiation response. TCAD simulations also suggest that the degradation is associated with a thermal spike generated by the synergy of the heavy-ion strike and applied bias voltage, with higher bias resulting in higher peak lattice temperatures, similar to a Rapid Thermal Anneal (RTA) at the MetalSemiconductor interface. This RTA at the MetalSemiconductor interface may result in inhomogeneity of the Schottky barrier associated with the $\mathrm{Ti} / 4 \mathrm{H}-\mathrm{SiC}$ contact [4]-[8].

\section{EXPERIMENTAL METHODS}

The devices used in this study were commercial SiC Schottky power diodes, manufactured by STMicroelectronics: STPSC1006D (600V, 10A) and STPSC 10H065DY $(650 \mathrm{~V}, 10 \mathrm{~A})$. The active areas for these diodes are estimated to be $2.31 \mathrm{~mm}^{2}$ and $2.62 \mathrm{~mm}^{2}$, respectively.

The diodes were de-capsulated to enable sufficient penetration of the heavy ions through the sensitive layers of the device (i.e., the Schottky junction and the epitaxial layer). The thickness of the $\mathrm{SiC}$ epitaxial layer is approximately $6 \mu \mathrm{m}$, hence the total thickness that the ions need to penetrate, including the overlying metal layers, was estimated to be on the order of ten micrometers. 
The heavy-ion irradiations were performed at the RADiation Effects Facility (RADEF) in the Accelerator Laboratory of the University of Jyväskylä [9]. The ion beams and their characteristics are tabulated in Table 1. All the irradiations were performed at room temperature and in vacuum conditions. All the device characterization was done at room temperature. The ion beams were at normal incidence to the device surface. The ion flux ranged between 20 and $6 \cdot 10^{5}$ ions. $\mathrm{cm}^{-2} \cdot \mathrm{s}^{-1}$ and cumulative fluences varied from $10^{3}$ to $10^{7}$ ions $\cdot \mathrm{cm}^{-2}$.

Table 1. Characteristics of the ion beams used at RADEF. The values for average LET and projected range are estimated using code from [10], described in [11].

\begin{tabular}{c|ccc}
\hline ION & $\begin{array}{c}\text { ENERGY } \\
{[\text { MeV] }}\end{array}$ & $\begin{array}{c}\text { LET(SiC) } \\
{\left[\mathbf{M e V} /\left(\mathbf{m g} / \mathbf{c m}^{2}\right)\right]}\end{array}$ & $\begin{array}{c}\text { Projected } \\
\text { Range } \\
{[\boldsymbol{\mu m}]}\end{array}$ \\
\hline $\mathrm{Xe}$ & 1217 & 62.4 & 60 \\
$\mathrm{Kr}$ & 768 & 33.8 & 63 \\
$\mathrm{Fe}$ & 523 & 20.1 & 64 \\
$\mathrm{Ar}$ & 372 & 10.9 & 78 \\
\hline
\end{tabular}

\section{RESULTS AND DISCUSSION}

When a sufficiently high reverse bias is applied to a SiC Schottky diode, increasing heavy-ion fluence, $\Phi$, increases the reverse leakage current. This effect was first reported in [3] and also in [2]. Figure 1 illustrates the evolution of leakage current during Xe-ion exposure for several different biasing conditions. The initial leakage current for all the tested devices at a reverse bias of $-200 \mathrm{~V}$ was in the range of 1 to $3 n A$. Higher biases during irradiation make the linear dependence of leakage current on the ion fluence more evident. In order for this gradual degradation to take place, the bias voltage must exceed a certain threshold, which depends on the ion and device characteristics. The data in Figure 1 were obtained for three different individual devices. Bias voltages between $-100 \mathrm{~V}$ and $-150 \mathrm{~V}$ represent one device, and biases $-180 \mathrm{~V}$ and $-200 \mathrm{~V}$ are for two separate devices.

As seen in Figure 1, for Xe-ions, the diode requires a bias voltage between $-130 \mathrm{~V}$ and $-140 \mathrm{~V}$ before onset of measurable degradation. Above this threshold, the rate of degradation increases with increasing bias. From the linear evolution of the leakage current as a function of ion fluence, one can extract the rate of degradation.

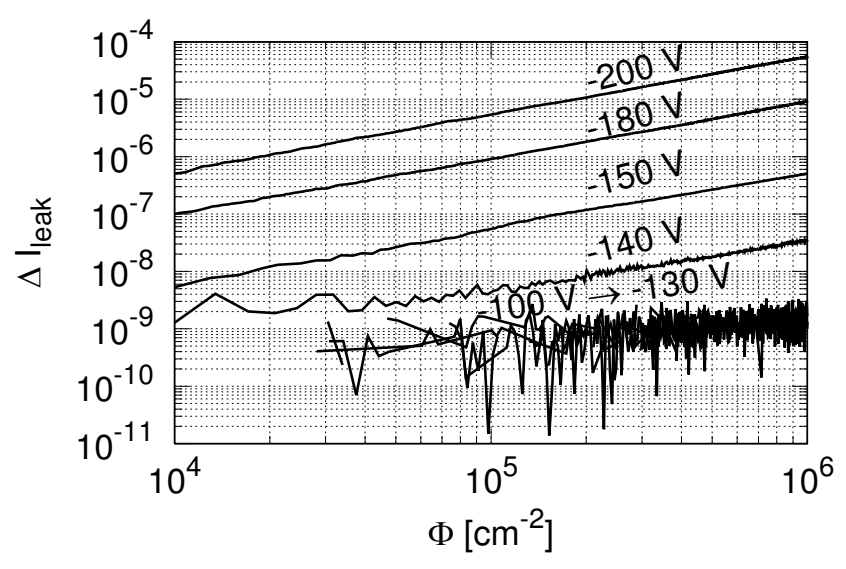

(b)

Figure 1. Leakage current in STPSC1006D devices as a function of Xe-ion fluence at different bias voltages applied during irradiation. Data taken from [12].

Figure 2(a) shows the evolution of the leakage current as a function of fluence measured at relatively low average ion flux of 45 ions $/ \mathrm{cm}^{2}$. These data demonstrate the Single Event Effect nature of the phenomenon. Each step in the leakage current data corresponds to a single ion strike. When measuring the leakage current evolution for the same ion at the same bias but at higher ion flux the integration time of the ammeter limits the observation of these steps. However, the average degradation rate is independent of the flux.

Moreover, not only the frequency of the ion-induced leakage steps is stochastic in nature, but also the magnitude of each step varies strongly depending on the ion and the applied bias. Whereas in Figure 2(a) the maximum step size, for $\mathrm{Xe}$ ion at bias of $-200 \mathrm{~V}$, is about $10 n A$, in case of $\mathrm{Ar}$ ions and bias of $-300 \mathrm{~V}$, shown in Figure 2(b) the same device type exhibits maximum step sizes up to $600 \mathrm{nA}$.

Figure 2(c) shows the current-voltage characteristics for a STPSC1006D diode measured after consecutive exposures to Xe-ions at fluence levels from $10^{3}$ to $10^{7}$ ions $/ \mathrm{cm}^{2}$. This data shows that the degradation of the whole reverse current-voltage curve is linearly proportional to the ion fluence. The deviation from this proportionality observed at low reverse voltage $\left(V_{\text {rev }}<\right.$ $50 \mathrm{~V}$ ) is due to noise floor in the current measurements set by the measurement equipment. This result has also been reported in [12], where the proportionality and the ion-modified current-voltage characteristics are discussed in detail. This linear proportionality suggests a simple test to determine usability for a specific environment: irradiate to a low fluence using the dominant ion species at the maximum reverse voltage required in the application. 

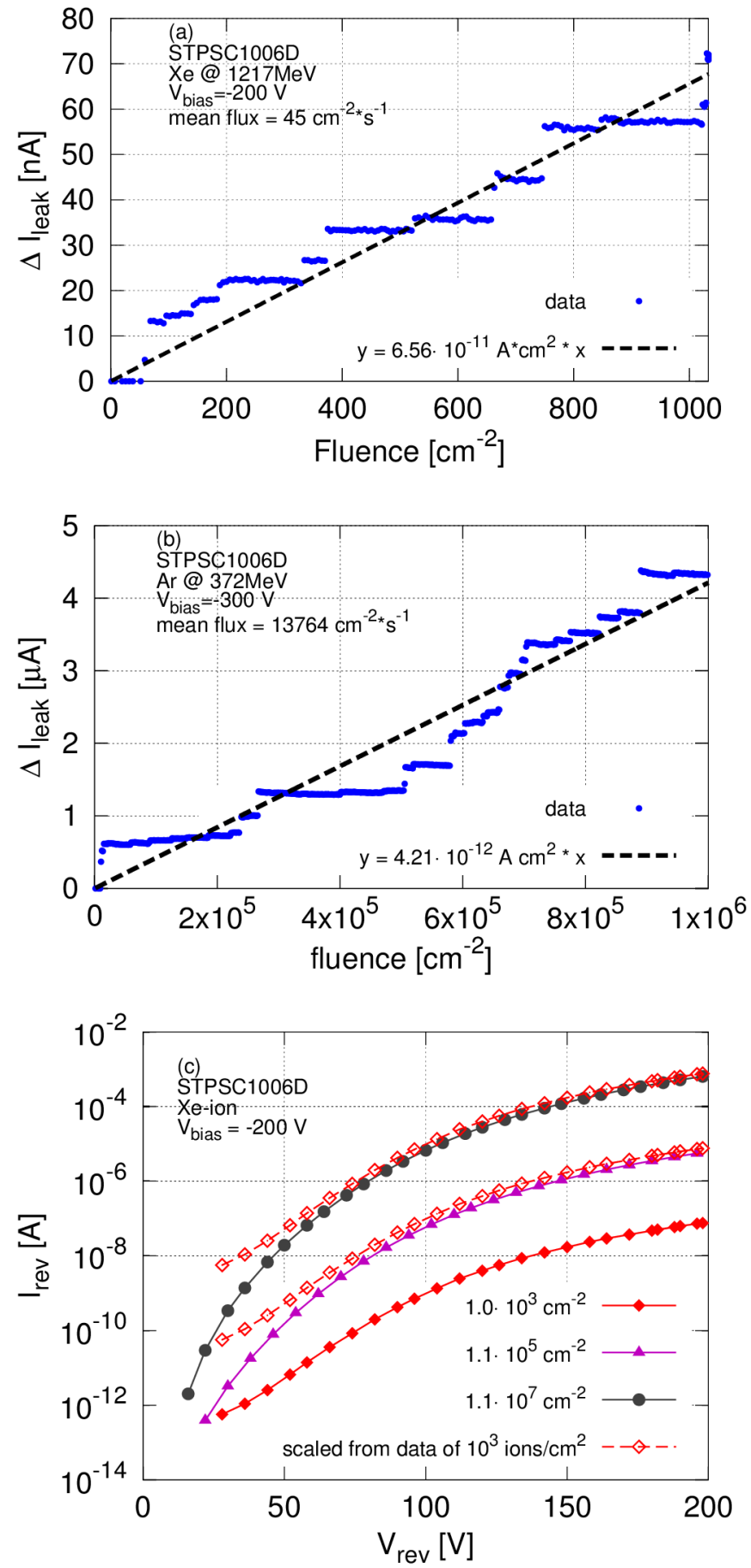

Figure 2. (a) The discrete incremental steps in leakage current induced by single $\mathrm{Xe}$ ions measured at bias of $-200 \mathrm{~V}$ at relatively low flux. (b) The discrete incremental steps in leakage current induced by single Ar ions measured at bias of $-300 \mathrm{~V}$ at relatively high flux. The average degradation rate is represented by the dashed line in both (a) and (b). (c) Experimental reverse current-voltage characteristics for the same device as in (a) irradiated at bias of $200 \mathrm{~V}$ with $\mathrm{Xe}$ ion fluences from $10^{3}$ to $10^{7}$ ions $/ \mathrm{cm}^{2}$. The measured degradation corresponding to a fluence of $10^{3}$ ions $/ \mathrm{cm}^{2}$ is scaled by factors corresponding to the fluence ratios, showing the proportionality of the ion-induced reverse leakage current to fluence.

Figure 3 presents the degradation rates as a function of reverse bias voltage during irradiation for both device types measured with four different ions. The graph shows that the rate of degradation first increases exponentially with increasing reverse bias during ion exposure. For Xe ions the rate saturates at higher biases. Also, the response is strongly dependent on the ion species. Both device types exhibit similar response.

The value for the degradation rate, $\Delta I / \Phi$, is given in units of $A \cdot \mathrm{cm}^{2}$. These values contain information about the probability for the heavy ion to induce the damage as well as the amount of damage in terms of leakage current.
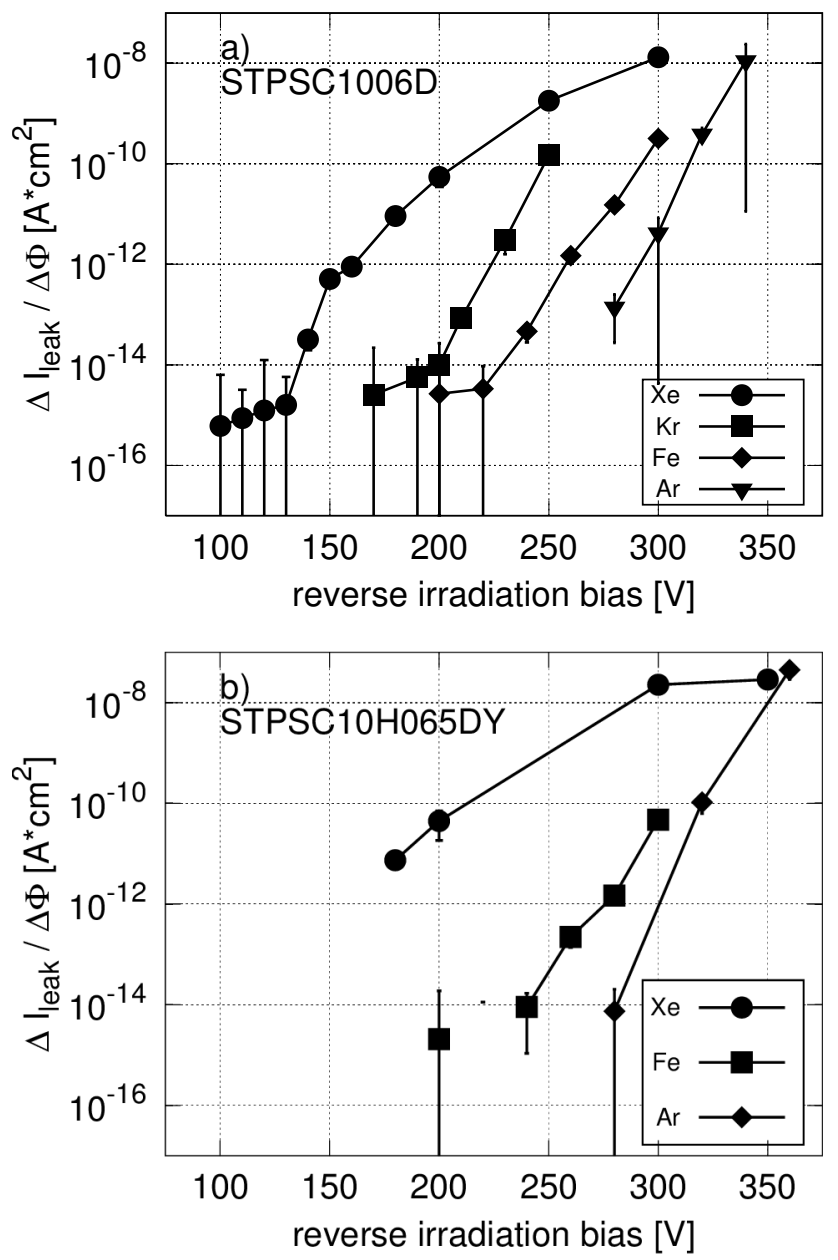

Figure 3. The rate of heavy-ion induced increase in leakage current during irradiation as a function of irradiation bias for STPSC1006D (a) and STPSC10H065DY (b) diodes and for different heavy ions. Error bars correspond to the $95 \%$ confidence interval of the standard error of regression obtained from data similar to Figure 2 (a) for each ion and bias configuration.

When a heavy ion traverses the semiconductor material, the energy lost by the ion goes mostly into generation of a dense cloud of electron-hole pairs along its trajectory. This cloud of excess charge carriers temporarily and locally modifies the conductivity of the semiconductor, reducing the resistivity at this location. When an electric field is applied in this region parallel to the ion's path, the reduced resistivity in this region 
allows prompt high current flow and heat dissipation due to Joule heating [3], [13], [14]. To illustrate this, VICTORY TCAD tools from Silvaco [15] were used. In Figure 4 and Figure 5 the TCAD simulation results are presented for temporal evolution of the maximum $\mathrm{SiC}$ lattice temperatures, and anode current after a Xe ion strike for two bias voltages applied to the Schottky structure, respectively. In these simulations the ion energy deposition in $\mathrm{SiC}$ is defined by the average LET value for $1217-\mathrm{MeV} \mathrm{Xe}$ ions, that is $62.4 \mathrm{MeV} /(\mathrm{mg} /$ $\mathrm{cm}^{2}$ ).

Already at $200 \mathrm{~V}$ reverse bias voltage, the ion strike causes a temperature rise, which locally goes above the melting temperature of $\mathrm{SiC}, 3000 \mathrm{~K}$. At a bias voltage of $350 \mathrm{~V}$, the temperature still recovers, although physical modification of the crystal is likely. This result is in agreement with results reported by Abbate et al. [13], [14]. The temperature values presented in Figure 4 should be used only as a proxy indicator for the assumed lattice damage caused by the combined effects of heavyion strikes and applied bias voltage. While the simulated lattice temperature is an imprecise measure of the local properties at very short times and small dimensions, it is indicative that there is a large amount of energy dissipated in a small volume. The higher energy dissipation at higher bias voltages could partly explain the increase in the rate of degradation at higher reverse biases, observed in Figure 3.

Schottky barrier engineering has been a topic of research for many years [4]-[8]. Annealing [6] and ion irradiation [7] have both been studied. The annealing temperatures examined are well within the temperatures TCAD simulations show at the metal (Ti) semiconductor $(4 \mathrm{H}-\mathrm{SiC})$ interface. These temperatures can vary in peak and in duration as the parameters for impact ionization and track charge distribution, both axial and radial, are varied. These track parameters, as well as effects associated with the anisotropy of material properties, are not well known for $4 \mathrm{H}-\mathrm{SiC}$, and remain topics of research [16]-[18]. What is consistent from simulations is that localized temperatures exceed $1000 \mathrm{~K}$, well above annealing temperatures. This localized high temperature could form Ti-silicide at the Ti-SiC interface locally lowering the Schottky Barrier height, resulting in a degree of inhomogeneity of the $\mathrm{Ti} / \mathrm{Ti}$ silicide Schottky barrier. This inhomogeneity gives the increase in reverse leakage current, which is proportional to fluence.

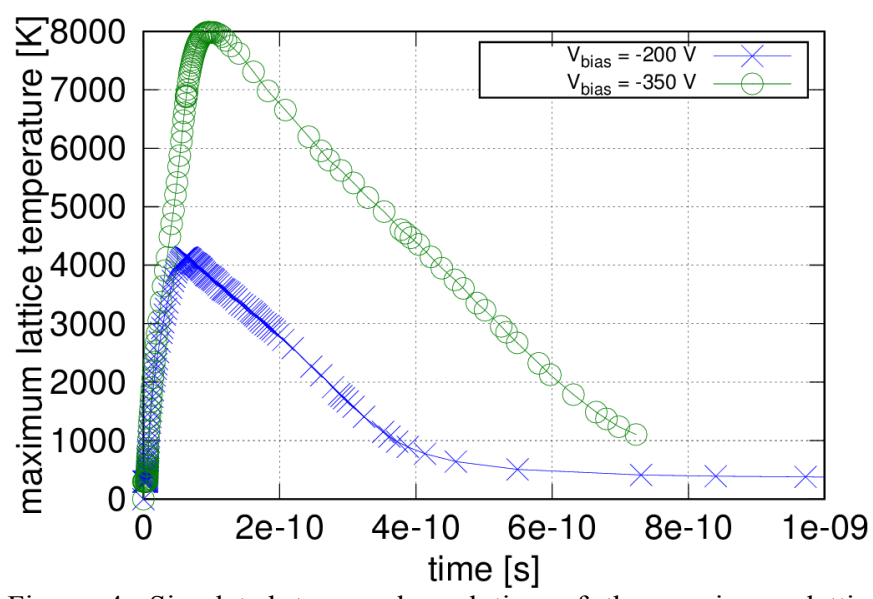

Figure 4. Simulated temporal evolution of the maximum lattice temperature in SiC Schottky diode structure after Xe ion strike. The average LET value of $62.4 \mathrm{MeV} /\left(\mathrm{mg} / \mathrm{cm}^{2}\right)$ is used for estimating the energy deposition by the strike. Different curves represent different reverse bias voltages applied on the Schottky diode.

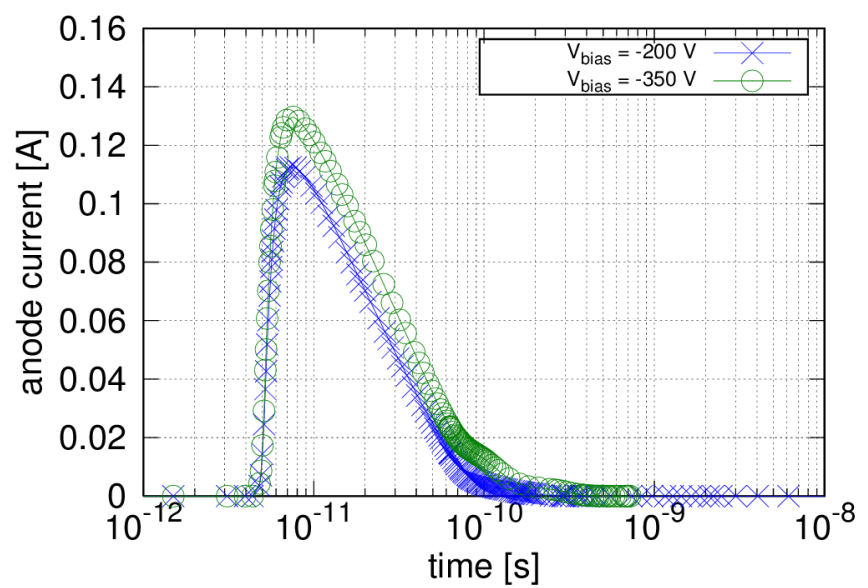

Figure 5. Simulated temporal evolution of the anode current after Xe ion strike in SiC Schottky diode structure. Different curves represent different reverse bias voltages applied on the Schottky diode.

Because the increase in the leakage current is observed only while a sufficient reverse bias is applied, the degradation can be attributed to ionization effects and not displacement damage. Usually the ionization effects of energetic heavy ions are described by the LET of the ions. However, the LET value describes only the average ionizing energy deposition per unit length for the impinging ion. For destructive or permanent effects, this is not always a valid metric[19], [20]. In the current case, the ion beam is partly scattered by the bonding wire attached on top of the diode. The scattered ions have different energy than the primary beam. Hence, they can deposit different amounts of energy in the target than that estimated from their original LET values. The effect of bonding wires on the radiation response in silicon power devices has been discussed in detail by Ferlet-Cavrois et al. [19]. In order to address this issue, Monte Carlo simulations for heavy-ion induced energy deposition in the depletion layer of a SiC Schottky diode 
have been performed using the Monte Carlo Radiative Energy Deposition (MRED) code [21]. The MRED simulations in this case take into account both direct ionization (i.e. LET) and indirect ionization (i.e. nonionizing energy loss, NIEL, and nuclear reactions) in the sensitive volume. However, in detailed analysis the effect of nuclear reactions and NIEL was negligible for the geometry and materials in question.

The thickness of the depletion region as a function of bias voltage, $V$, can be estimated by using

$$
W=\sqrt{\frac{2 \varepsilon_{s}}{q N_{D}}\left(V_{b i}-V-\frac{k T}{q}\right)},
$$

where $N_{D}$ is the doping level of the epilayer, $\varepsilon_{S}$ is the permittivity of $\mathrm{SiC}, q$ is the elementary charge, and $k T$ is the thermal energy factor [22]. The doping level of the epilayer for these devices was estimated from $\mathrm{C}-\mathrm{V}$ measurements to be $N_{D} \approx 10^{16} \mathrm{~cm}^{-3}$. The thickness of the depletion region was used in the Monte Carlo simulations as the sensitive layer where the energy deposition from the ions occurs. For the diodes under study, the epilayer thickness is about $6 \mu \mathrm{m}$, which means that full depletion is achieved at reverse voltage of about $-325 \mathrm{~V}$.

The presence of the bonding wire was also taken into account in the simulations. The thickness of the wire was estimated to be about $400 \mu \mathrm{m}$. Wire deformation was taken into account by using a cylinder with elliptical cross-section. The structure used in the energy deposition simulations is shown in Figure 6. The dimensions for the wire are $300 \mu \mathrm{m}$ and $533 \mu \mathrm{m}$ for thickness and width, respectively. The length of the wire was estimated to shadow $25 \%$ of the diode area.

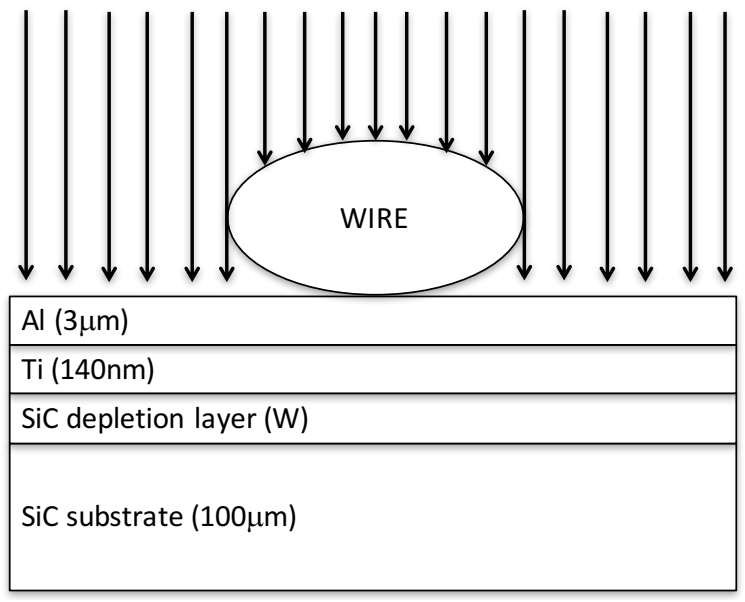

Figure 6. Structure used in energy deposition simulations. The thickness of the depletion layer, , $W$, is estimated by using Eq.(1).

In the following, the heavy-ion induced degradation is attributed to a thermal spike similarly as in ref [3], which to first order is estimated from the peak power dissipated at the ion strike location. The peak power is estimated to be

$$
P_{\text {peak }} \propto \frac{\Delta E}{\Delta x} \cdot V_{\text {rev }}^{2}
$$

where $\Delta E / \Delta x$ corresponds to the actual energy deposited per unit length in the depletion layer. By using this approach instead of average values of LET [3], the simulation results are better correlated with the experimental data. In this approach, a critical value for peak power, $P_{\text {crit }}$, was set. At this point only qualitative correlation between simulated and experimental results is expected for different ions.

Figure 7 presents the complementary cumulative distribution functions (CCDF) for the peak power events obtained from simulations for structures with and without the bonding wire for all used ions. The results for the other ions show similar characteristics, but the effect of the wire is the strongest for Ar ions. The graphs also show the estimated critical value for the peak power, $P_{\text {crit }}$. The magnitude of the degradation is assumed to be the same for all events above the critical peak power.

From these spectra by taking the probability for the events to exceed the critical peak power, we can estimate these probabilities as a function of bias voltage for all the ions. This is presented in Figure 8. These results clearly show the effect of the bonding wire. The probabilities for Ar ions to exceed critical peak power without the bonding wire are extremely low. Whereas with the bonding wire, the probabilities show similar characteristics as in the experimental results given in Figure 3. For Xe ions the probability for the simulated peak power to exceed the critical value saturates, which also corresponds to the effect observed experimentally. However, in the experimental data the saturation is not as distinct as in the simulation results. This difference could be partly explained by the difference in the thermal spike response illustrated in Figure 4. At higher reverse bias voltages, the lattice peak temperature reaches higher values, resulting in higher degradation rates. 

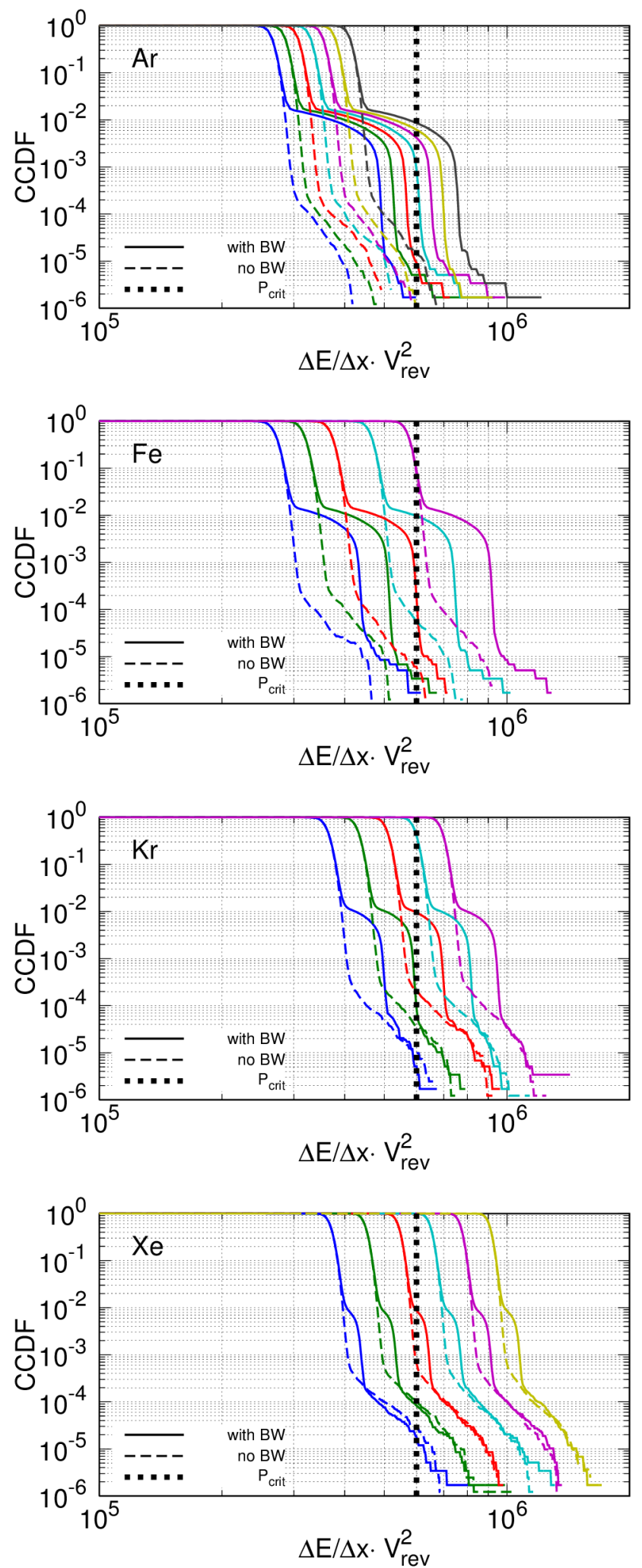

Figure 7. Complementary cumulative distribution functions (CCDF) for the peak powers for all the used ions simulated with (solid) and without (dashed) the bonding wire. The dashed vertical line represents the estimated critical peak power, $P_{\text {crit }}$. The reverse bias increases in the curves from left to right.

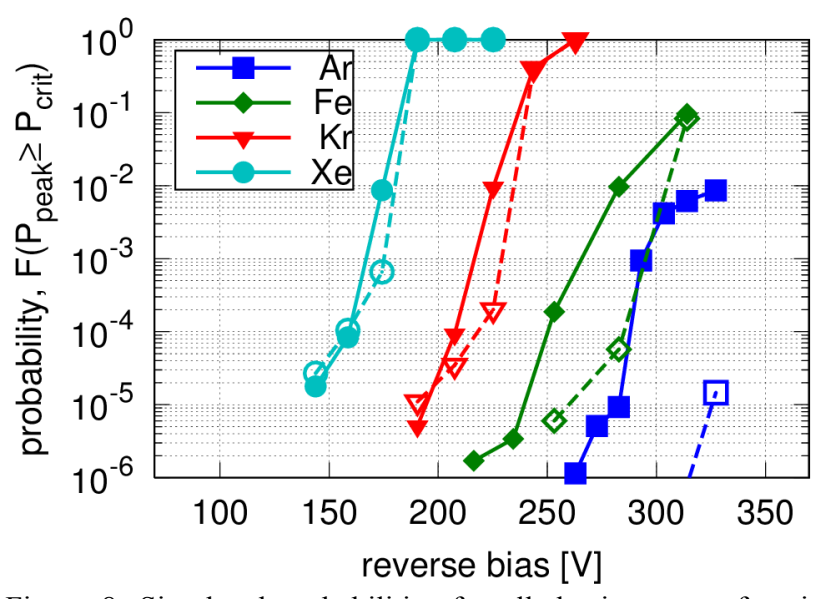

Figure 8. Simulated probabilities for all the ions as a function of reverse bias. Probability represent the case in which power production in the depletion region of the $\mathrm{SiC}$ Schottky diode exceeds the critical peak power, $P_{\text {crit }}$. Filled and open symbols correspond to the simulations with and without the bonding wire, respectively.

These results suggest that in heavy-ion tests for $\mathrm{SiC}$ Schottky diodes the observed degradation is not correlated with the bias and the average energy deposition in the depletion region as given by the LET, as proposed in [3]. However, it was found that in order for the degradation to occur, a certain peak power as defined by the energy deposition of an individual ion in the diode depletion layer and the bias must be reached. This is is described in Eq.(2). This means that for ions with lower LET, such as the Ar ions considered here, only the events of ionizing energy deposition that are at the high end of the energy deposition spectrum can contribute to the degradation when sufficient bias is applied.

These results can also be used to estimate the on-orbit behavior of these SiC Schottky diodes. For geosynchronous orbit, CRÈME-2009 [23] estimates an isotropic flux of about $5.4 \cdot 10^{-2}$ ions $/\left(\mathrm{cm}^{2} \cdot\right.$ day $)$ for ions with LET above $20 \mathrm{MeV} /\left(\mathrm{mg} / \mathrm{cm}^{2}\right)$, i.e. the maximum LET of the Ar ions considered here. Assuming typical derating, these SiC Schottky diodes might be used at an operation voltage of $\left|V_{\max }\right|=$ $350 \mathrm{~V}$. Taking the degradation rate from Figure 3, we get about $3 \cdot 10^{-8} \mathrm{~A} \cdot \mathrm{cm}^{2}$ at this level of reverse bias for all ions used in this work. Hence, taking these values, and assuming degradation to be independent of the direction of the impinging ions, the worst case increase in leakage current in these diodes during a one-year mission in GEO is about $600 \mathrm{nA}$. One needs to keep in mind that this value assumes that all ion-induced damage events to be equal. But due to the stochastic nature of ion-induced damage this amount (or more) of damage can be reached after a single (or a few) ion strike(s) as is demonstrated in Figure 2(b). 


\section{CONCLUSIONS}

Heavy-ion induced reverse leakage current increase in SiC Schottky power diodes is demonstrated to be due to the synergistic effects of energy deposition by the ions and the bias voltage. This degradation is possibly related to thermal spot annealing at the metal semiconductor interface. This thermal annealing leads to an inhomogeneity of the Schottky barrier that shows a strong correlation to the increase in leakage current as a function of fluence. The physical construction of the device including the bonding wires, a form of shielding, affects the results. Detailed Monte Carlo and TCAD simulations were used to get better understanding of the heavy-ion induced degradation in $\mathrm{SiC}$ diodes in terms of ion energy deposition and bias. The results suggest that at lower bias voltages not all ions (even those at high LET) hitting the diode area contribute to the degradation. The degradation occurs only when a sufficient bias is applied and the impinging ion generates enough excess charge carriers in the depletion region to enable high peak power dissipation in a very small volume, via Joule heating, at the strike location leading to permanent damage. This is confirmed by TCAD simulations, which also show a bias dependence, in which higher reverse bias voltages produce higher peak temperatures in the $\mathrm{SiC}$ Schottky diode after the ion strike. The proportionality to the ion fluence of the experimentally measured degradation allows testing at low fluence and then scaling to determine the amount of time a device can operate in an environment before reverse leakage current exceeds design requirements.

\section{ACKNOWLEDGEMENTS}

The authors want to thank Dr. Derek Kimpton and Dr. Marek Turowski of Silvaco for their useful discussions and support in simulation. The help from Dr. Heikki Kettunen, Mikko Rossi and Jukka Jaatinen of RADEF facility at University of Jyväskylä is gratefully acknowledged. Authors are also thankful for Dr. Andrew Woodworth of NASA Glenn Research Center for the physical analysis on the devices.

\section{REFERENCES}

[1] S. Kuboyama, C. Kamezawa, Y. Satoh, T. Hirao, and H. Ohyama, "Single-Event Burnout of Silicon Carbide Schottky Barrier Diodes Caused by High Energy Protons," IEEE Trans. Nucl. Sci., vol. 54, no. 6, pp. 2379-2383, Dec. 2007.

[2] E. Mizuta, S. Kuboyama, H. Abe, Y. Iwata, and T. Tamura, "Investigation of Single-Event Damages on Silicon Carbide (SiC) Power MOSFETs," IEEE Trans. Nucl. Sci., vol. 61, no. 4, pp. 1924-1928, Aug. 2014.

[3] S. Kuboyama, C. Kamezawa, N. Ikeda, T. Hirao, and H. Ohyama, "Anomalous Charge Collection in Silicon Carbide Schottky Barrier Diodes and Resulting Permanent Damage and Single-Event Burnout," IEEE Trans. Nucl. Sci., vol. 53, no. 6, pp. 3343-3348, Dec. 2006.

[4] D. Defives, O. Noblanc, C. Dua, C. Brylinski, M. Barthula, V. Aubry-Fortuna, and F. Meyer, "Barrier inhomogeneities and electrical characteristics of $\mathrm{Ti} / 4 \mathrm{H}-\mathrm{SiC}$ Schottky rectifiers," IEEE Trans. Electron Devices, vol. 46, no. 3, pp. 449-455, Mar. 1999.

[5] L.-C. Han, H.-J. Shen, K.-A. Liu, Y.-Y. Wang, Y.-D. Tang, Y. Bai, H.-Y. Xu, Y.-D. Wu, and X.-Y. Liu, "Annealing temperature influence on the degree of inhomogeneity of the Schottky barrier in Ti/4H-SiC contacts," Chinese Phys. B, vol. 23, no. 12, p. 127302, Dec. 2014.

[6] L. Calcagno, A. Ruggiero, F. Roccaforte, and F. La Via, "Effects of annealing temperature on the degree of inhomogeneity of nickel-silicide/SiC Schottky barrier," $J$. Appl. Phys., vol. 98, no. 2, p. 23713, 2005.

[7] F. Roccaforte, S. Libertino, F. Giannazzo, C. Bongiorno, F. La Via, and V. Raineri, "Ion irradiation of inhomogeneous Schottky barriers on silicon carbide," J. Appl. Phys., vol. 97, no. 12, p. 123502, 2005.

[8] G. Pristavu, G. Brezeanu, M. Badila, R. Pascu, M. Danila, and P. Godignon, "A model to non-uniform Ni Schottky contact on $\mathrm{SiC}$ annealed at elevated temperatures," Appl. Phys. Lett., vol. 106, no. 26, p. 261605, Jun. 2015.

[9] A. Virtanen, H. Kettunen, A. Javanainen, M. Rossi, and J. Jaatinen, "RADiation Effects Facility at JYFL." [Online]. Available: http://www.jyu.fi/accelerator/radef.

[10] A. Javanainen, "European Component Irradiation Facilities Cocktail Calculator,” 2015. [Online]. Available: http://research.jyu.fi/radef/ECIFcalc/.

[11] A. Javanainen, "A simple expression for electronic stopping force of heavy ions in solids," Nucl. Instruments Methods Phys. Res. Sect. B Beam Interact. with Mater. Atoms, vol. 285, pp. 158-161, Aug. 2012.

[12] A. Javanainen, K. F. Galloway, V. Ferlet-Cavrois, J.-M. Lauenstein, F. Pintacuda, R. D. Schrimpf, R. A. Reed, and A. Virtanen, "Charge Transport Mechanisms in Heavy-Ion Driven Leakage Current in Silicon Carbide Schottky Power Diodes," IEEE Trans. Device Mater. Reliab., vol. 16, no. 2, pp. 208-212, Jun. 2016.

[13] C. Abbate, G. Busatto, P. Cova, N. Delmonte, F. Giuliani, F. Iannuzzo, A. Sanseverino, and F. Velardi, "Analysis of Heavy Ion Irradiation Induced Thermal Damage in $\mathrm{SiC}$ Schottky Diodes," IEEE Trans. Nucl. Sci., vol. 62, no. 1, pp. 202-209, Feb. 2015.

[14] C. Abbate, G. Busatto, P. Cova, N. Delmonte, F. Giuliani, F. Iannuzzo, A. Sanseverino, and F. Velardi, "Thermal damage in SiC Schottky diodes induced by SE heavy ions,"

Microelectron. Reliab., vol. 54, no. 9-10, pp. 2200-2206, Sep. 2014.

[15] Silvaco Inc., "Victory Device 3D Device Simulator." [Online]. Available: http://www.silvaco.com/products/tcad/device_simulation/vic tory_device/victory_device.html.

[16] K. Bertilsson, H.-E. Nilsson, and C. S. Petersson, "Simulation of anisotropic breakdown in $4 \mathrm{H}-\mathrm{SiC}$ diodes," in COMPEL 2000. 7th Workshop on Computers in Power Electronics. Proceedings (Cat. No.00TH8535), pp. 118-120.

[17] T. Hatakeyama, T. Watanabe, T. Shinohe, K. Kojima, K. Arai, and N. Sano, "Impact ionization coefficients of $4 \mathrm{H}$ silicon carbide," Appl. Phys. Lett., vol. 85, no. 8, p. 1380, 2004.

[18] T. Hatakeyama, "Measurements of impact ionization coefficients of electrons and holes in $4 \mathrm{H}-\mathrm{SiC}$ and their 
application to device simulation," Phys. status solidi, vol. 206, no. 10, pp. 2284-2294, Oct. 2009.

[19] V. Ferlet-Cavrois, J. R. Schwank, S. Liu, M. Muschitiello, T. Beutier, A. Javanainen, A. Hedlund, C. Poivey, A. Mohammadzadeh, R. Harboe-Sorensen, G. Santin, B. Nickson, A. Menicucci, C. Binois, D. Peyre, S. K. Hoeffgen, S. Metzger, D. Schardt, H. Kettunen, A. Virtanen, G. Berger, B. Piquet, J.-C. Foy, M. Zafrani, P. Truscott, M. Poizat, and F. Bezerra, "Influence of Beam Conditions and Energy for SEE Testing," IEEE Trans. Nucl. Sci., vol. 59, no. 4, pp. 1149-1160, Aug. 2012.

[20] M. A. Xapsos, "Applicability of LET to single events in microelectronic structures," IEEE Trans. Nucl. Sci., vol. 39, no. 6, pp. 1613-1621, 1992.

[21] R. A. Weller, M. H. Mendenhall, R. A. Reed, R. D. Schrimpf, K. M. Warren, B. D. Sierawski, and L. W. Massengill, "Monte Carlo Simulation of Single Event Effects," IEEE Trans. Nucl. Sci., vol. 57, no. 4, pp. 17261746, Aug. 2010.

[22] S. M. Sze, Physics of Semiconductor Devices. New York: John Wiley and Sons, Inc., 1981.

[23] B. Sierawski and M. H. Mendenhall, "CREME-MC site." [Online]. Available: https://creme.isde.vanderbilt.edu/. 